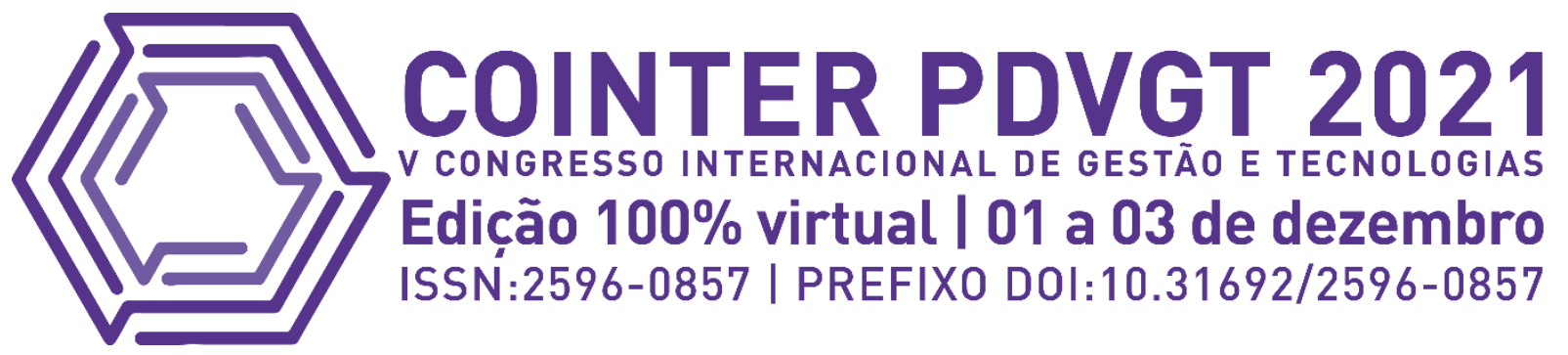

\title{
SISTEMA DE IDENTIFICAÇÃO VEICULAR PARA MONITORAMENTO DE TRÁFEGO
}

\section{SISTEMA DE IDENTIFICACIÓN DE VEHÍCULOS PARA EL SEGUIMIENTO DEL TRÁFICO}

\section{VEHICLE IDENTIFICATION SYSTEM FOR TRAFFIC MONITORING}

\author{
Apresentação: Comunicação Oral \\ Wolker Sanches Dias ${ }^{1}$; André Quintiliano Bezerra Silva ${ }^{2}$
}

DOI: https://doi.org/10.31692/2596-0857.VCOINTERPDVGT.0101

\section{RESUMO}

As fronteiras brasileiras são alvos de atuação de criminosos no contrabando de drogas e roubos de carros devido a um efetivo reduzido de policiais, além de possuir uma área muito extensa para ser fiscalizada. Boa parte das drogas, armas e demais contrabandos que entram no Brasil são transportados por veículos terrestres e saem da Bolívia ou Paraguai com destino aos estados de São Paulo e/ou Rio de Janeiro. Uma maneira de aumentar a fiscalização e ajudar os órgãos de segurança pública no combate desses e outros crimes é com a utilização de tecnologias para monitorar o tráfego de veículos nas estradas brasileiras. Devido aos últimos avanços na área de inteligência artificial, em especial na área de visão computacional permitiu o desenvolvimento de sistemas de reconhecimento de placas de veículos (RPV). No entanto, os métodos tradicionais são razoavelmente eficazes sob circunstâncias específicas ou apenas em suposições fortes, e há poucos bancos de dados para detecção de placas de carros. A tarefa de reconhecimento de placas possui dificuldades como, ângulo, formas, cores, padrões e iluminação não uniforme no momento da captura das imagens. Essa pesquisa desenvolveu um sistema de reconhecimento automático de placas de veículos empregando o algoritmo YOLOv3 para para identificar e reconhecer caracteres. Embora como esse algoritmo ainda sofre com alguns problemas, um novo método de detecção de placa de carro em tempo real baseado no YOLOv3 melhorado foi proposto. O modelo atingiu $95 \%$ de precisão no reconhecimento de caracteres considerando as placas antigas e as novas do Mercosul.

Palavras-Chave: sistema rpv, redes neurais convolucionais, detecção de objetos, vigilância de tráfego

\section{ABSTRACT}

The Brazilian borders are targets of criminal activity in drug smuggling and car theft due to a reduced number of police officers, in addition to having a very large area to be inspected. Much of the drugs, weapons and other contraband entering Brazil are transported by land vehicles and leave Bolivia or

\footnotetext{
${ }^{1}$ Licenciatura em Computação, Instituto Federal de Mato Grosso do Sul, wolker.sd@hotmail.com

${ }^{2}$ Programa de Pós-Graduação em Ciências da Computação, Universidade Federal de Mato Grosso do Sul, andre.bezerra@ifms.edu.br
} 


\section{SISTEMA DE IDENTIFICAÇÃO VEICULAR PARA MONITORAMENTO}

Paraguay with destination in the states of São Paulo and/or Rio de Janeiro. One way to increase inspection and help public security agencies fight these and other crimes is to use technologies to monitor vehicular traffic on Brazilian roads. Due to the latest advances in the field of artificial intelligence, especially in the area of computer vision, it allowed the development of license plate recognition systems (RPV). However, traditional methods are reasonably effective under specific circumstances or only strong assumptions, and there are few databases for license plate detection. The license plate recognition task has difficulties such as angle, shapes, colors, patterns and non-uniform lighting when capturing images. This research developed an automatic license plate recognition system employing the YOLOv3 algorithm to identify and recognize characters. Although as this algorithm still suffers from some problems, a new real-time license plate detection method based on the improved YOLOv3 has been proposed. The model achieved 95\% accuracy in character recognition considering the old and new Mercosur plates.

Keywords: rpv system, convolutional neural networks, object detection, traffic surveillance.

\section{INTRODUÇÃO}

As fronteiras brasileiras são alvos de atuação de criminosos no contrabando de drogas e roubos de carros que se dão devido a um efetivo reduzido de policiais e uma área muito extensa a ser fiscalizada. Boa parte das drogas e contrabandos que entram no Brasil são transportadas em rodovias por veículos terrestres que partem da Bolívia ou Paraguai com destino aos estados de São Paulo e Rio de Janeiro.

O ex-diretor da Polícia Federal que combate o crime organizado, Oslain Campos Santana, disse em sua apresentação na subcomissão no Senado Federal contra o tráfico de drogas, que os vizinhos brasileiros (Colômbia, Peru e Bolívia) são os maiores produtores de drogas do mundo. Ainda na apresentação, Santana comentou que boa parte da produção das drogas bolivianas e colombianas entram no Brasil através do Paraguai.

Para se ter uma dimensão das fragilidades fronteiriças que o Brasil possui, basta observar a fronteira americana com o México. Entre eles, há uma faixa terrestre de aproximadamente 3 mil quilômetros, e é altamente vigiada por policiais e com diversos dispositivos tecnológicos, além de uma série de muros e cercas que impedem cruzamentos ilegais entre os dois países. Mesmo com todo esse aparato de defesa, ainda assim, os traficantes conseguem transportar toneladas de drogas para as cidades norte-americanas. No Brasil, além de contar com um baixo efetivo de policiais federais e/ou estaduais, mal equipados e treinados, ainda deixam de contar com a ajuda de tecnologias existentes ao seu favor para o combate ao crime (COSTA, 2017).

Devido a essas e outras fragilidades, os criminosos aproveitam para vender ao Brasil, drogas, armas, cigarros, entre outros contrabandos. No balanço divulgado no site Campo 
Grande News, de janeiro a abril de 2020, a Polícia Militar Ambiental (PMA), já apreendeu no estado do Mato Grosso do Sul (MS), 37,4 toneladas de drogas, principalmente maconha. No mesmo período de 2019 foram 16,3 toneladas. De janeiro a abril de 2018 foram 19,4 toneladas apreendidas pela PMA e 17,6 no mesmo período de 2017. Outros dados de apreensões de drogas pela Polícia Federal, em conjunto com a Polícia Rodoviária Federal mostra o grande número de entorpecentes entrando em terras sul-mato-grossenses com principal destino, os estados de São Paulo e Rio de Janeiro. Até o fim de agosto de 2019, no MS, o total de cocaína apreendida já somava mais que o dobro no mesmo período do ano de 2018 .

Além do tráfico, também existe um mercado de veículos roubados no Brasil que são legalizados nesses mesmos países e que crescem a cada ano e de forma alarmante. No Paraguai, o Congresso Nacional criou uma lei em 2007 que permite legalizar carros de origem não comprovada ou duvidosa (GAZETA DO POVO, 2007).

Uma maneira de aumentar a fiscalização e ajudar as polícias no combate desses e outros crimes é implementar a utilização de tecnologias para monitorar o tráfego de veículos nas rodovias, principalmente os sistemas de monitoramento com reconhecimento de placas.

Existem muitos sistemas de reconhecimento de placas no mercado, sendo que estes ou utilizam técnicas antigas que demoram na identificação, ou necessitam de condições quase ideais para que a extração e identificação dos caracteres funcionem corretamente.

A área de reconhecimento de placas de veículos (RPV) tem sido um grande problema de visão computacional nas últimas décadas e está sendo uma ferramenta importante no controle dos veículos em diversos países. A tarefa de gerenciar o tráfego terrestre é bastante desafiadora, pois exige desse tipo de sistemas uma capacidade de reconhecer veículos e placas em diversos cenários. Segundo (BULAN, 2017), os sistemas tradicionais de RPV geralmente possuem três estágios: detecção de placas, segmentação de caracteres e reconhecimento de caracteres. O primeiro estágio exige uma maior precisão, pois, uma falha na detecção da placa do veículo provavelmente leva ao erro nos próximos estágios. No cenário do mundo real, as imagens são capturadas durante em um ambiente altamente complexo, como baixa iluminação, distância de câmera e ângulo ruim, além de condições climáticas que podem influenciar bastante na qualidade da captura dos veículos.

Alguns algoritmos tradicionais para a deteção e tradução da objetos com texto, já foram implementados, entre elas estão: o AdaBoost (Zhang 2010), que é rápido, mas pode 


\section{SISTEMA DE IDENTIFICAÇÃO VEICULAR PARA MONITORAMENTO}

falhar se a distância da placa do carro for muito grande, o algoritmo de detecção baseado em recursos de textura (Hanzo, 1999) e (Wu, 2013) que tem a maior precisão nas condições de alto contraste, textura clara, o algoritmo de detecção de morfologia matemática (Kim 2002) e (Jin, 2011) possui boa velocidade de identificação, mas precisa ser combinado com outros algoritmos de detecção. Além dessas soluções, ainda foram desenvolvidos o algoritmo de detecção baseado em célula não linear (Zhang, 2010) e o algoritmo de detecção baseado em segmentação de imagens coloridas (Zhang, 2015) e (Abolghasemi, 2009), ambos com boa precisão, embora lentos. Esses algoritmos são conhecidos pelos seus métodos tradicionais de detecção de carros, mas possuem grandes limitações para serem utilizados nas diferentes cenas do mundo real (Wazalwar, 2013).

Com o surgimento do aprendizado profundo, o estado da arte começou a se mover para outra direção. Hoje em dia muitos trabalhos empregam CNNs devido à sua alta precisão para detecção e reconhecimento genérico de objetos, como observado no trabalho de (ZHANG, 2016). Além desse modelo, outros foram criados utilizando a rede CNN como base, entre eles pode-se destacar: R-CNN (Girshick, 2014), Fast R-CNN (Girshick, 2015), Faster-RCNN (Girshick, 2015). Esses modelos são baseados na classificação e possuem uma maior precisão, embora perdem no tempo de detecção. Já os que utilizam regressão, como Yolo (Redmon, 2016), SSD (Liu, 2016), YOLOv2 (Redmon, 2017), YOLOv3 (Redmon, 2018) são mais rápidos e podem atingir bons resultados em tempo real, mas possuem menor precisão.

Embora haja vários modelos de RPV disponíveis na literatura, ainda existe espaço para melhorar a detecção e/ou a extração dos caracteres. A partir desta visão, este artigo apresenta um modelo RPV baseado em deep learning usando um YOLOv3 para detecção das placas, além de outro modelo neural para melhorar a precisão de extração dos caracteres. $\mathrm{O}$ modelo proposto tem um total de quatro processos principais: pré-processamento, localização e detecção das placas veiculares, segmentação de caracteres através de uma CNN. O modelo foi validado experimentalmente usando um conjunto de dados de referência, e o resultado da simulação confirmou que o modelo apresentado produziu bons resultados.

As partes restantes desta pesquisa estão organizadas da seguinte forma. A Fundamentação Teórica que discute sobre as teorias, a Metodologia que mostra a natureza e os métodos utilizados. Por fim, serão mostrados os resultados e as conclusões. 


\section{FUNDAMENTAÇÃO TEÓRICA}

A identificação automática de placas de veículos de forma eficiente, sem a necessidade de intervenção humana tem se tornado cada vez mais importante nos últimos anos. Existem várias razões pelas quais a necessidade de identificação aumentou. No cenário recente, há um número crescente de carros circulando e todos eles possuem placas. $\mathrm{O}$ rápido desenvolvimento da tecnologia de processamento de imagens tornou possível identificar e detectar placas de veículos em alta frequência.

A identificação de placas de veículos é útil para muitas empresas privadas e órgãos públicos. As agências governamentais podem localizar carros e outros veículos que estão envolvidos em um crime, verificar se os impostos e multas estão pagos ou identificar o proprietário do veículo que infringe as leis de trânsito. Muitos países como EUA, Japão, Alemanha, Itália, Reino Unido e França aplicaram e implementaram o RPV com sucesso em sua gestão de tráfego. Vários operadores privados também podem se beneficiar dos sistemas $\mathrm{RPV}$, como, em estacionamentos privados. O objetivo principal do sistema RPV é reconhecer a placa veicular em imagem ou vídeo, procurá-la em um banco de dados e registrar alguma informação sobre o carro. O requisito para um sistema PRV é alta precisão ao ler as placas e também tempo de processamento rápido.

A dificuldade em reconhecer as placas dos veículos nos diferentes conjuntos de teste afetará a precisão do sistema, fazendo comparações diretas da precisão e sem considerar a complexidade, não faz sentido. Conforme apontado pelos autores, não é apropriado declarar qual método apresenta o melhor desempenho devido à falta de formas uniformes de avaliá-lo.

Um sistema RPV típico pode ser dividido em duas fases principais:

1. Detecção da placa do veículo

2. Segmentação de caracteres - extraia os caracteres alfanuméricos da placa

3. Reconhecimento de dígitos - reconhece cada dígito individual na placa.

Cada estágio foi implementado usando várias técnicas de aprendizado de máquina. As técnicas tradicionais de aprendizado de máquina incluem recursos escolhidos por humanos para representar os recursos subjacentes da imagem. 


\section{SISTEMA DE IDENTIFICAÇÃO VEICULAR PARA MONITORAMENTO}

\section{Redes neurais convolucionais}

Uma Rede Neural Convolucional (ConvNet / CNN) é um algoritmo de Aprendizado Profundo que pode receber dados de uma ou mais dimensões, atribuir (pesos e bias) aos objetos de entrada, como por exemplo imagens e ter como resultado final a capacidade de diferenciar cada objeto. O pré-processamento exigido em uma CNN é muito menor em comparação com outros algoritmos de classificação. Enquanto nos métodos primitivos os filtros são projetados à mão, com treinamento suficiente, as CNNs têm a capacidade de aprender esses filtros Em outras palavras, os filtros nada mais são, que características extraídas das entradas.

Há vários tipos diferentes de CNNs, como Googlenet, Resnet, Alexnet, VGG etc. Existem basicamente dois modelos de aprendizagem durante a execução de tarefas de processamento de imagem. Eles são chamados de aprendizagem supervisionada e não supervisionada. Aprendizagem supervisionada refere-se à aprendizagem por meio de entradas pré-rotuladas, que atuam como alvos. O objetivo do treinamento supervisionado é reduzir o erro de classificação do modelo, por meio do cálculo correto do valor de saída do exemplo de treinamento. Aprendizagem não supervisionada significa que contém um conjunto de treinamento que não contém nenhum rótulo de saída e são mais comumente usados para agrupar os dados. Uma rede neural convolucional consiste em uma camada de entrada e uma de saída, bem como em várias camadas ocultas dentro dela. As camadas ocultas consistem em um CNN normalmente consistem em camadas convolucionais, camadas de agrupamento (pooling), camadas totalmente conectadas (fully connected). Cada camada tem uma função específica e elas são explicadas a seguir.

\section{Camada de convolução}

A camada de convolução é o principal bloco de construção de uma rede neural convolucional. Conforme vamos mais fundo em outras camadas de convolução, os filtros estão fazendo produtos pontuais para a entrada das camadas de convolução anteriores. A convolução é a primeira camada a extrair recursos de uma imagem de entrada. A convolução preserva a relação entre os pixels, aprendendo os recursos da imagem usando pequenos quadrados de dados de entrada. É uma operação matemática que leva duas entradas, como matriz de imagem e um filtro ou kernel. 


\section{Camada de pooling}

As camadas de pool permitem reduzir o número de parâmetros quando as imagens são muito grandes. O agrupamento espacial também chamado de subamostragem ou amostragem reduzida, que reduz a dimensionalidade de cada mapa, mas retém as informações importantes. O agrupamento espacial pode ser de diferentes tipos, como agrupamento de soma, de agrupamento máximo e agrupamento médio. Max pooling e Average pooling tomam o maior elemento dos mapas de características, enquanto o segundo, faz a média dos valores. A soma total de todos os elementos em um mapa de recursos é conhecida como Sum pooling.

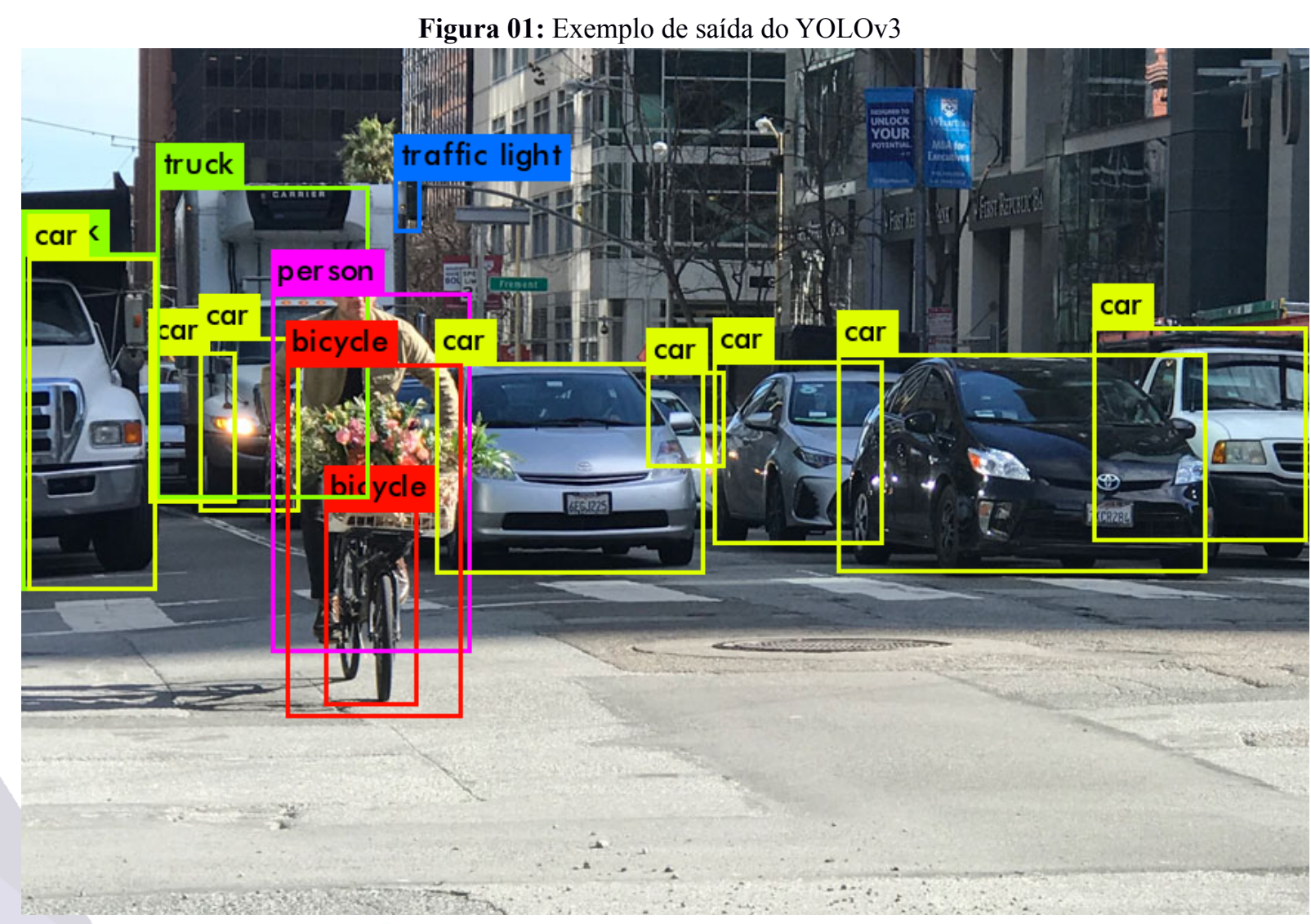

\section{Camada totalmente conectada}

Fonte: Própria (2019).

Após várias camadas de convolução e preenchimento, precisaríamos da saída na forma de uma classe. As camadas de convolução e pooling seriam capazes apenas de extrair recursos e reduzir o número de parâmetros das imagens originais. No entanto, para gerar a saída final, precisamos aplicar uma camada totalmente conectada para gerar uma saída igual ao número de classes de que precisamos. Torna-se difícil atingir esse número apenas com as camadas de convolução. Camadas de convolução geram mapas de ativação 3D enquanto nós apenas precisamos da saída para saber se uma imagem pertence ou não a uma classe particular. 


\section{SISTEMA DE IDENTIFICAÇÃO VEICULAR PARA MONITORAMENTO}

\section{YOLOv3}

YOLOv3 (You Only Look Once, Versão 3) é um algoritmo de detecção de objetos em tempo real que identifica objetos específicos em vídeo ou imagens. YOLO usa recursos aprendidos por uma rede neural convolucional profunda para detectar um objeto. As versões 1-3 do YOLO foram criadas por Joseph Redmon e Ali Farhadi. A primeira versão do YOLO foi criada em 2016, e a versão 3, que é discutida nesta pesquisa, foi feita dois anos depois, em 2018.

Os sistemas de classificação de objetos são usados por programas de Inteligência Artificial (IA) para perceber objetos específicos em uma classe como assuntos de interesse. Os sistemas classificam os objetos em imagens em grupos onde os objetos com características semelhantes são colocados juntos, enquanto outros são negligenciados, a menos que programados para fazer o contrário. Como típico para detectores de objetos, os recursos aprendidos pelas camadas convolucionais são passados para um classificador que faz a previsão de detecção. No YOLO, a previsão é baseada em uma camada convolucional que usa convoluções $1 \times 1$. YOLO é denominado "você olha apenas uma vez" porque sua previsão usa convoluções $1 \times 1$; o tamanho do mapa de previsão é exatamente o tamanho do mapa de feições antes dele.

YOLO é uma Rede Neural Convolucional (CNN) para realizar a detecção de objetos em tempo real. CNNs são sistemas baseados em classificadores que podem processar desde de séries temporais de 1 dimensão até dados 3D, embora sejam amplamente utilizadas para identificar padrões entre elas, como pode ser visto na Figura 1. YOLO tem a vantagem de ser muito mais rápido do que outras redes e ainda mantém a precisão.

Ele permite que o modelo veja a imagem inteira no momento do teste, de forma que suas previsões sejam informadas pelo contexto global da imagem. O YOLO e outros algoritmos de rede neural convolucional "classificam" regiões com base em suas semelhanças com classes predefinidas. As regiões com pontuação alta são notadas como detecções positivas de qualquer classe com a qual se identificam mais intimamente. Por exemplo, em um vídeo de tráfego ao vivo, o YOLO pode ser usado para detectar diferentes tipos de veículos, dependendo de quais regiões do vídeo têm alta pontuação em comparação com classes predefinidas de veículos. 
Figura 02: YOLOv3 é executado muito mais rápido do que outros métodos de detecção.

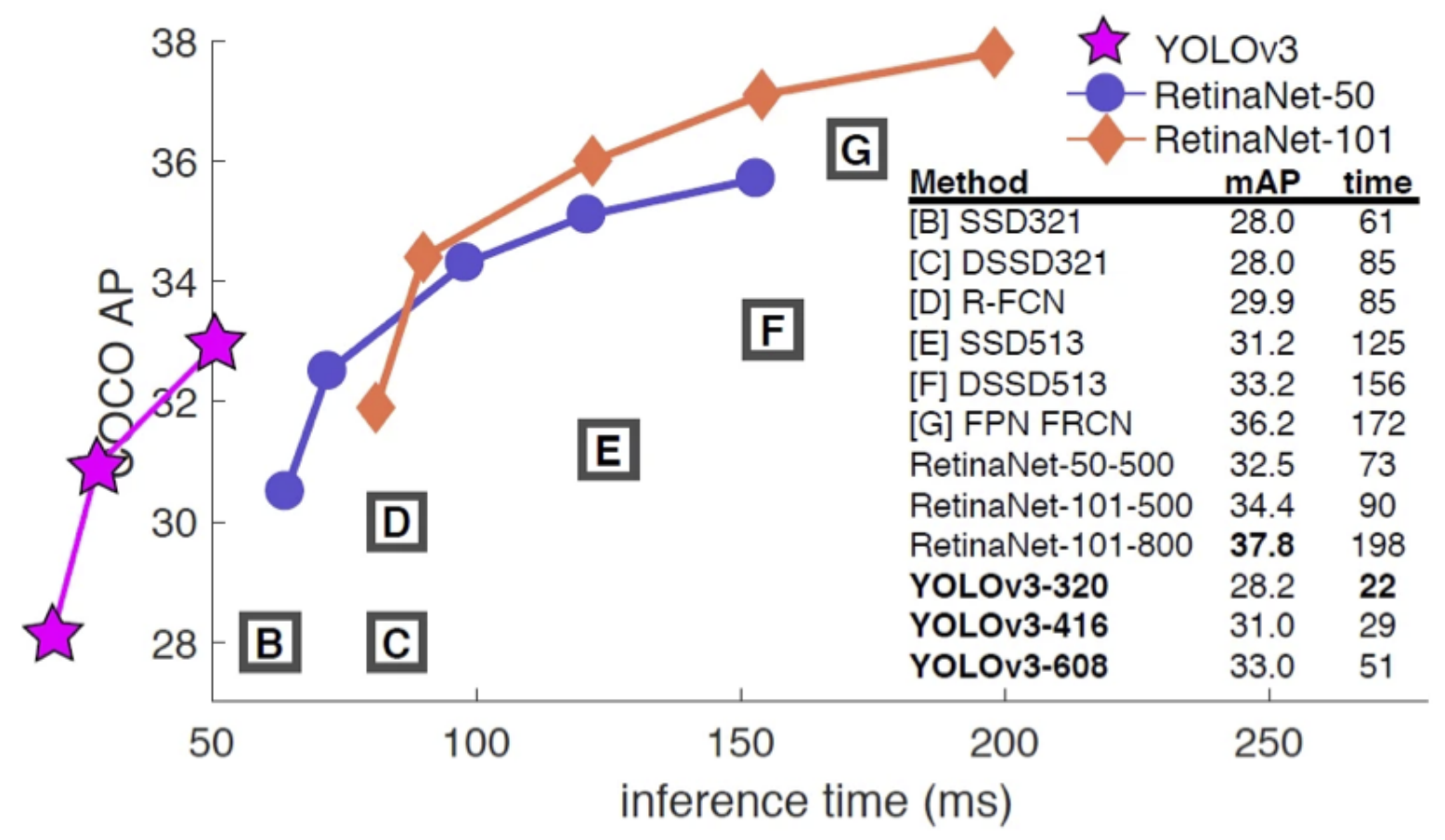

Fonte: Própria (2019).

YOLOv3 usa Darknet-53, que é um backbone também feito pelos criadores do YOLO. A Darknet-53 tem 53 camadas convolucionais em vez das 19 anteriores, tornando-o mais poderoso do que Darknet-19 e mais eficiente do que backbones concorrentes (ResNet-101 ou ResNet-152).

Usando o gráfico fornecido no artigo YOLOv3 de Redmon e Farhadi, pode-se ver que Darknet-52 é 1,5 vezes mais rápido do que ResNet101. A precisão representada não envolve qualquer compensação entre precisão e velocidade entre os backbones Darknet, uma vez que ainda é tão preciso quanto o ResNet-152, mas duas vezes mais rápido.

YOLOv3 é rápido e preciso em termos de precisão média (mAP) e valores de intersecção sobre união (IOU) também. Ele é executado significativamente mais rápido do que outros métodos de detecção com desempenho comparável (daí o nome - Você só olha uma vez).

Além disso, é possível ver facilmente trocar entre velocidade e precisão simplesmente alterando o tamanho do modelo, e nenhum retreinamento necessário, como pode ser visto na Figura 2. 


\section{SISTEMA DE IDENTIFICAÇÃO VEICULAR PARA MONITORAMENTO}

\section{METODOLOGIA}

A classificação dessa pesquisa utilizou como base os critérios apresentados por Vergara (1998), que qualifica a pesquisa em relação a duas vertentes: quanto aos fins e quanto aos meios. Quanto aos fins, consistiu em uma pesquisa conclusiva, pois é um tipo de pesquisa que testa hipóteses e examina relações específicas entre variáveis.

Quanto aos meios, trata-se de uma pesquisa experimental e bibliográfica. Conforme Vergara (1998), esse tipo de investigação é recomendada quando o pesquisador manipula e controla variáveis independentes e observa as variações que tal manipulação e controle produzem em variáveis dependentes. Ela também pode ser considerada bibliográfica porque foi desenvolvida utilizando exclusivamente fontes bibliográficas acessíveis ao público em geral, como livros, artigos, teses e dissertações. E a natureza investigativa deste trabalho é uma pesquisa quantitativa.

\section{Base de dados}

Dados significam muito na era da aprendizagem profunda, normalmente as pesquisas precisam de dados para treinar seu modelo. Embora a detecção de placas de carros tenha se desenvolvido há muito tempo, ainda falta um banco de dados padrão. A maior parte do trabalho é baseada no banco de dados dos próprios pesquisadores, portanto torna-se difícil uma comparação entre os trabalhos nessa área específica. Além disso, a base de dados disponibilizada ao público também é bastante limitada. Para esse trabalho foram recolhidos 3000 imagens de diferentes tipos de veículos brasileiros. As imagens foram obtidas de diferentes formas, através de câmeras, telefones celulares e na internet. Ao capturar imagens, consideramos diferentes condições, como variação nas condições de iluminação e ângulo de visão e presença de vários veículos e placas na mesma imagem. Também foram capturados diferentes tipos de veículos, incluindo motocicletas, ônibus, caminhões, etc. Algumas características do conjunto de dados são as seguintes:

- Para garantir a variação das condições de iluminação, coletamos imagens de placas de veículos durante o dia, no crepúsculo e à noite. Também coletamos imagens de locais onde a alta variação de iluminação está presente.

- Diferentes tipos de veículos no Brasil têm pequenas diferenças no formato da placa em termos de cor e tamanho. Para capturar essa variabilidade, diferentes tipos de imagens de veículos foram obtidas. Os principais veículos que cobrimos são carro, motocicleta, ônibus, 
caminhão e van coberta.

- Consideramos as placas localizadas tanto na frente quanto na traseira de um veículo.

- Uma tendência comum é ter uma única placa de carro por imagem. Mas, isso não é adequado para detecção de placas de veículos em tempo real. Nosso conjunto de dados contém várias placas em uma única imagem, que é importante para treinar e testar um sistema de detecção em tempo real.

Figura 03: Amostra de imagens do nosso conjunto de dados de veículos coletados.
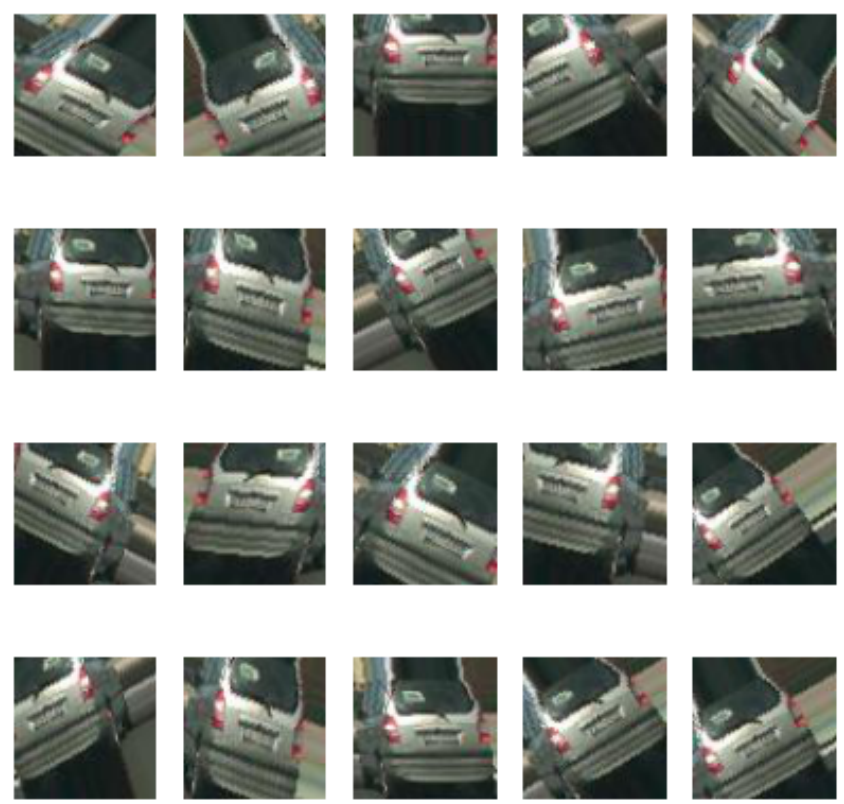

Fonte: Própria (2019).

A maioria dos sistemas de detecção de placa possuem câmeras estáticas, e em certas condições não podem lidar com as inúmeras possibilidades que acabam aparecendo no cenário do mundo real. Para simular o cenário do mundo real, capturamos imagens de diferentes ângulos de visão. Devido à multiplicidade de condições, as imagens capturadas podem ser ruins e o sistema de detecção deve ser robusto o suficiente para detectá-las também. O conjunto de dados contém imagens borradas, com pouca luminosidade e de baixa qualidade.

Para cada um dos 36 caracteres que uma placa pode possuir foi criado um conjunto de dados de caracteres contendo 2.112 imagens para cada um deles, totalizando 76.032 imagens. As imagens sintéticas foram geradas com letras de $\mathrm{A}$ à $\mathrm{Z}$ e números de 0 a 9 nas fontes Mandatory e FE Engschrift que correspondem respectivamente às placas antigas (cinzas) e novas (Mercosul). Alguns exemplos de caracteres são mostrados na Figura. 4. 


\section{SISTEMA DE IDENTIFICAÇÃO VEICULAR PARA MONITORAMENTO}

Figura 04: Imagens de amostra de nosso conjunto de dados de caracteres.

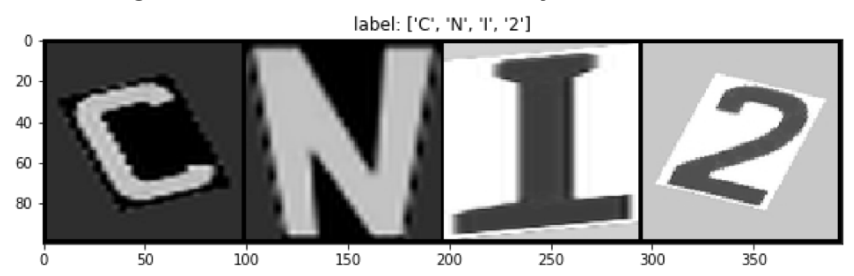

Fonte: Própria (2019).

Figura 05: Pipeline da proposta de rede de três estágios para reconhecimento de placas de veículos. No primeiro estágio, o YOLOv3 foi usado para detectar e localizar placas de veículos no conjunto de dados coletados. No segundo estágio, todos os dígitos e caracteres foram reconhecidos. Na etapa final, uma rede CNN foi usada para reconhecer cada letra e número segmentados.

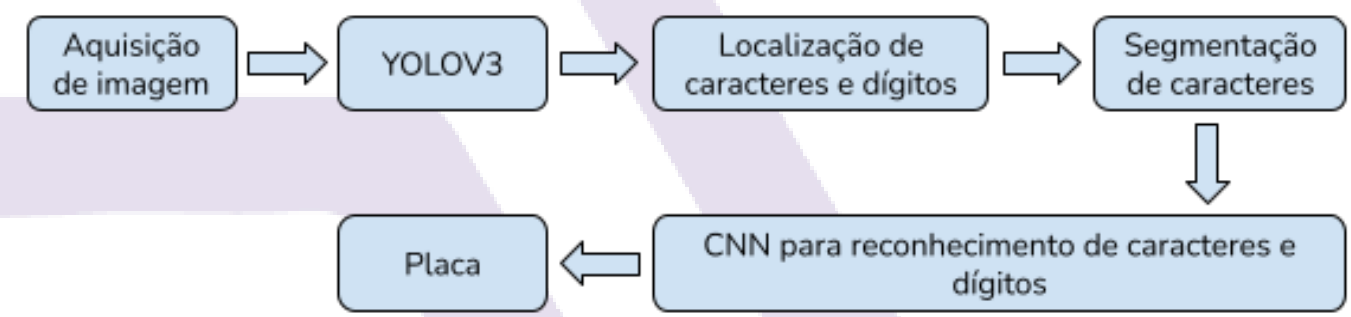

Fonte: Própria (2021).

\section{Modelo neural}

O modelo proposto foi dividido em três estágios: detecção e localização de placas de veículos, reconhecimento dos dígitos e identificação de caracteres e reconhecimento de caracteres. A Figura 5 mostra o pipeline do método proposto. No primeiro estágio utilizou-se o algoritmo YOLOv3 para detectar e localizar os veículos e suas respectivas placas. Depois disso, as placas foram cortadas para cada veículo encontrado na imagem. No estágio seguinte, uma $\mathrm{CNN}$ foi utilizada para detectar e classificar os 36 dígitos possíveis que podem aparecer em uma placa brasileira.

\section{A. Detecção e localização da placa}

A detecção e localização de placas de veículos é uma tarefa crucial nos sistemas de detecção de placas. Alguns trabalhos procuram identificar apenas as placas, enquanto outros preferem primeiro detectar o veículo antes de detectar a placa. Em nosso caso, devido à multiplicidade do tipo de veículo e à diferença na localização da placa do veículo, a detecção do veículo é de suma importância. Assim, tanto a frente do veículo, como a traseira dele são detectadas, além da placa.

Para detectar o veículo e a placa, o algoritmo YOLOv3 foi empregado (Redmon, 
2018). YOLOv3 não é a versão mais recente do algoritmo YoLo, porém, consegue obter bons resultados em comparativo com as novas versões, (Redmon, 2019). A base de dados começou com 4500 imagens e, após uma limpeza nessa base, algumas imagens foram eliminadas, pois estavam repetidas ou com qualidade inferior ao que se desejava. No final, foram obtidas 3000 imagens. Desse total, $80 \%$ das imagens foram usadas para treinamento e os outros $20 \%$ para validação. Para a marcação das caixas delimitadoras, a ferramenta web LabelBox foi utilizada. Essa ferramenta permite criar as classes do problema, delimitar e exportar a base de dados no formato $\mathrm{COCO}$, que é padrão utilizado para treinamento da YoLo. Antes do treinamento, algumas configurações foram aplicadas no algoritmo do YOLOv3 para torná-lo adequado para a detecção de placas. O tamanho do batch foi definido como 64. As subdivisões foram definidas como 16 para um treinamento mais rápido. $\mathrm{Na}$ fase de detecção da placa, tínhamos apenas uma classe e, como tal, tomamos o tamanho do kernel igual a 18. Pesos pré-treinados para as camadas convolucionais darknet-53 foram usados como pesos iniciais para o treinamento. O modelo foi iterado 300 vezes.

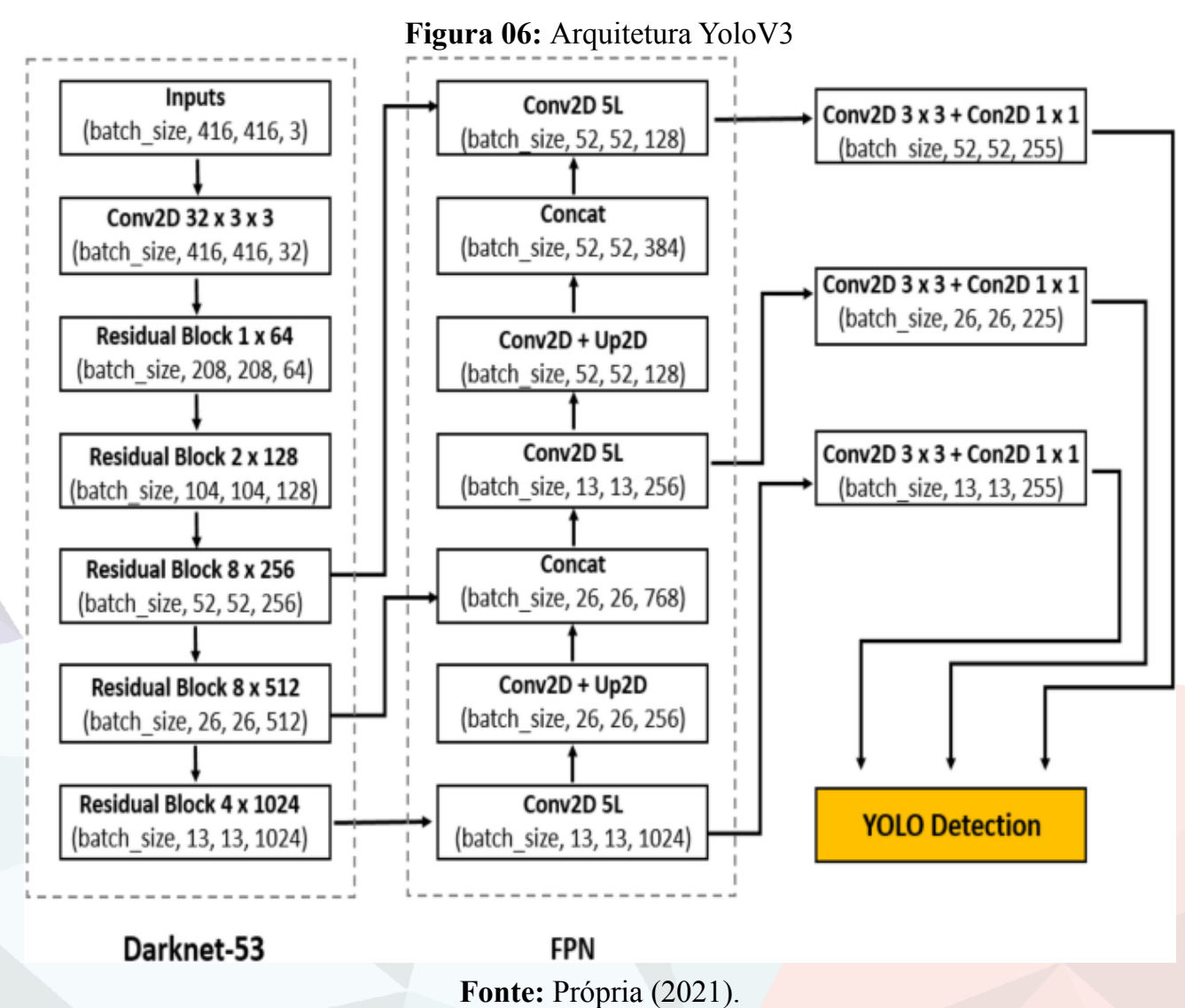




\section{SISTEMA DE IDENTIFICAÇÃO VEICULAR PARA MONITORAMENTO}

\section{B. Reconhecimento de dígitos e caracteres}

A partir da coordenada da caixa delimitadora fornecida pela rede YOLOv3, as placas foram segmentadas mais uma vez com o auxílio da ferramenta web LabelBox. As caixas delimitam as regiões que contêm cada um dos caracteres. Neste estágio, foi aplicado data augmentation por rotação aleatória, alteração de contraste e adicionando ruído gaussiano.

Como a placa brasileira tem 7 dígitos (variando de 0 a 9) e caracteres de $\mathrm{A}$ à $\mathrm{Z}$, essa técnica permitiu aumentar o número de caracteres em quatro vezes. Nessa etapa, os caracteres ainda não são reconhecidos pelo modelo, apenas identificados as localizações deles na placa. Para isso, projetamos uma rede CNN customizada para detectar 36 classes (10 dígitos e 26 caracteres). Novamente, $80 \%$ das imagens foram usadas para treinamento e o restante foi usado para validação. Como a resolução da nossa imagem era boa, não foi necessário alterar o tamanho da entrada na rede como feito por (Montazzolli, 2018). Em vez disso, o preenchimento em escala de cinza para garantir que estamos alimentando a imagem de tamanho fixo na rede. Mantendo a proporção da imagem inalterada, redimensionamos cada imagem para $96 \times 96$ por meio de preenchimento.

Figura 07: Algumas das amostras previstas de reconhecimento de dígitos e identificação de caracteres de nosso método proposto. Vê-se que em (a) - (d), inclinação, rotação, borrão, etc. não estão restringindo o sistema a reconhecer dígitos e identificar caracteres.

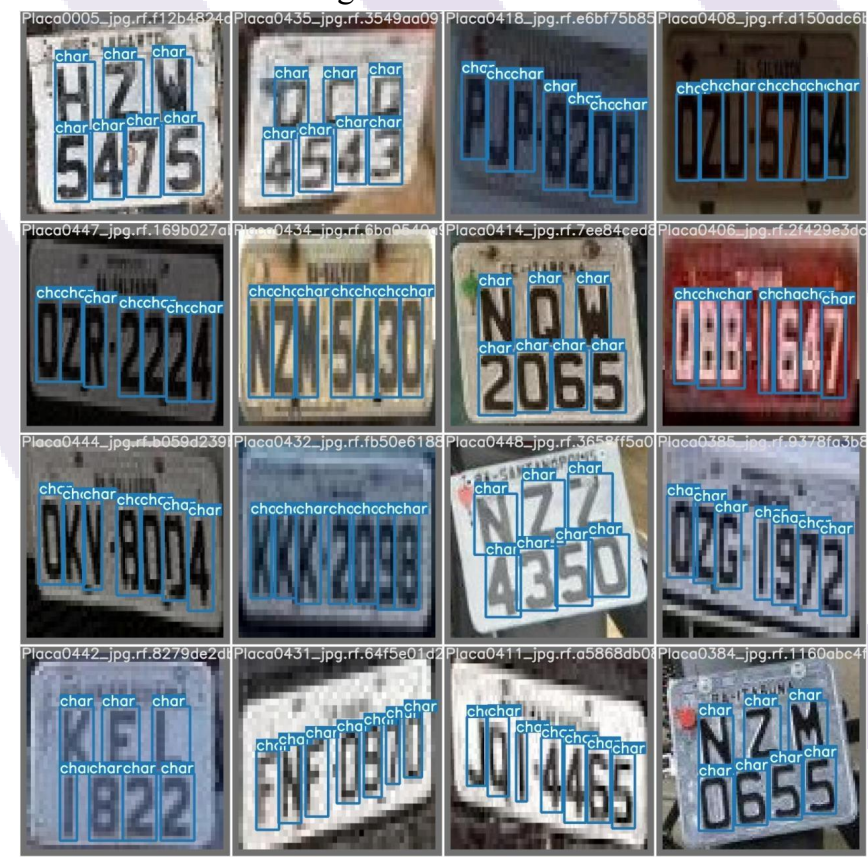

Fonte: Própria (2021).

A rede CNN foi treinada usando o otimizador Adam, e a função de loss, a entropia cruzada por 100 épocas. Foram testadas várias taxas de aprendizado começando com $10^{-3} \mathrm{e}$ 
elas foram reduzidas gradualmente. A Tabela I resume as configurações de hiperparâmetros adotados na rede proposta. Verificou-se que a rede proposta estava ficando superdimensionada, devido à alta capacidade de aprendizado do modelo. Portanto, houve a necessidade de adicionar uma normalização em lote (batch norm) após cada camada convolucional, exceto após a última função de ativação ReLU, para reduzir o sobreajuste e aumentar o desempenho do modelo.

\section{RESULTADOS E DISCUSSÃO}

Neste artigo, todos os experimentos foram conduzidos no banco de dados adquirido. Foram definidas 2600 imagens para treinamento e 400 amostras para validação. $\mathrm{O}$ ambiente experimental utilizado foi o Colab Google, que possui GPU para os testes. Os parâmetros iniciais do modelo são definidos da seguinte forma: uma taxa de aprendizado de $10^{-3}$, tamanho do lote: 64, número de épocas: 300 e o otimizador Adam. Neste artigo, a métrica IOU é utilizada para julgar se a previsão é precisa ao comparar o resultado previsto do modelo e o alvo. Quando IOU > 0,5, é julgado como uma previsão precisa, caso contrário, é julgado como falsa previsão.

Verdadeiro Positivo (VP) é o número de placas perfeitamente identificadas. Verdadeiro Negativo (VN) é o número de placas não perfeitamente detectadas. Falso positivo (FP) é o número de imagens detectadas incorretamente como positivas que, na verdade, não são placas. Falso negativo $(\mathrm{FN})$ é o número de não placas detectadas incorretamente que são realmente placas.

Quando o modelo é usado para detecção da placa do veículo, a precisão representa a precisão de previsão do modelo na placa do veículo. Recall representa a placa do carro não detectada do modelo. Alguns resultados são apresentados na Figura 7.

A partir da Figura 7, o modelo proposto completa a tarefa de detecção de forma considerável. Na Figura 7 pode-se observar diferentes situações na imagem. Como clima diferente, luz insuficiente, distância de tiro diferente. Os resultados mostram que o modelo tem bons resultados. O modelo não apenas detecta a posição da placa do carro, mas também obtém um alto valor de confiança. 
SISTEMA DE IDENTIFICAÇÃO VEICULAR PARA MONITORAMENTO

Tabela 01: Resultados da detecção da placa do carro

\begin{tabular}{cccccc}
\hline VP & FP & VN & FN & Precision & Recall \\
2700 & 55 & 41 & 4 & $95 \%$ & $95,21 \%$ \\
\hline
\end{tabular}

Fonte: Adaptado de Dados fictícios, apenas para fins ilustrativos (2020).

Como visto na Tabela 1, o modelo detecta a maioria das placas do carro. Ele falha em algumas imagens desafiadoras que estão sob condições de desfoque e muito brilhantes. Em conclusão, o modelo proposto pode detectar a placa do veículo satisfatoriamente.

Como comparação, ainda foi executado o banco de dados no YOLOv3 original. Conforme listado na Tabela 2, embora ambos os métodos possam detectar a placa do veículo, o modelo proposto é melhor do que o YOLOv3 original.

Tabela 02: Resultados da detecção da placa do carro

\begin{tabular}{ccccccc}
\hline Modelo & VP & FP & VN & FN & Precision & Recall \\
YOLOv3 & 2541 & 98 & 56 & 12 & $90 \%$ & $88,21 \%$ \\
Modelo & 2700 & 25 & 41 & 4 & $95 \%$ & $95 \%$ \\
\hline
\end{tabular}

Fonte: Adaptado de Dados fictícios, apenas para fins ilustrativos (2020).

Como pode ser visto na Tabela 2, o YOLOv3 original tem menor precisão e recall. O modelo pode lidar com o banco de dados de forma mais eficaz. Quanto menos perda de informações, melhores serão os resultados. A Fig. 8 mostra exemplos da saída da fase proposta de reconhecimento de dígitos e identificação de caracteres. Nosso método proposto reconhece corretamente os dígitos em, enquanto o método falhou em reconhecer alguns dos dígitos devido à alta inclinação no ângulo de visão e, em alguns casos, transparência dos dígitos e caracteres.

Figura 08: Exemplo de reconhecimento

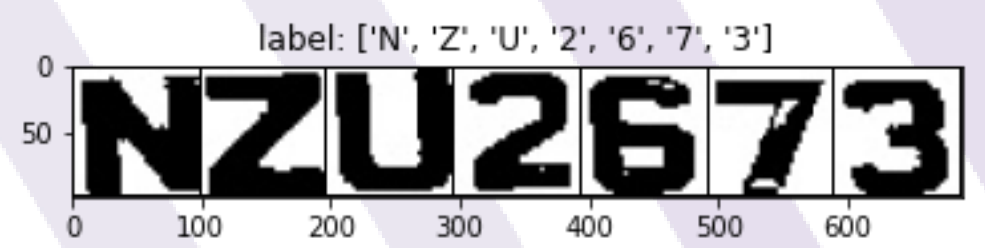

Fonte: Própria (2021). 


\section{CONCLUSÕES}

Nesta pesquisa, uma abordagem precisa, altamente eficiente e robusta é proposta para localizar e reconhecer placas de veículos brasileiros nas regiões de fronteira do Brasil. Um conjunto de dados desafiador de placas antigas (cinzas) e novas (Mercosul) foi apresentado. $\mathrm{Na}$ era do aprendizado profundo, um banco de dados com diversidade suficiente poderia estimular o desempenho do sistema, embora não seja o caso. Neste trabalho, foi utilizado um banco de dados de placas de carros estabelecido pelos autores. O banco de dados com placas veiculares inclui diferentes situações. Como ângulo, imagens com maior claridade ou muito escuro. Todas elas ajudam a treinar o modelo. $\mathrm{O}$ foco deste trabalho é propor um modelo melhorado baseado em YOLOv3. Para não perder muitas informações da imagem, o tamanho da entrada foi expandido. Por meio das operações de concatenação e aumentar a amostragem, o modelo pode fundir diferentes dimensões de mapas de recursos. A fusão dos diferentes mapas de características dimensionais faz com que o modelo obtenha informações em várias escalas. Nos resultados experimentais, o modelo aprimorado pode detectar a placa do carro melhor do que o Yolov3 original. Não importa o efeito visual ou o índice de avaliação, o modelo proposto acabou se saindo melhor. $\mathrm{O}$ modelo proposto não apenas detectou com precisão a placa, mas também extraiu corretamente os caracteres.

Pretendemos, no futuro, adicionar mais imagens ao nosso conjunto de dados atual dos países que fazem fronteira com o Brasil para desenvolver um sistema de reconhecimento de placas em tempo real de última geração. 


\section{REFERÊNCIAS}

COSTA, MAURÍCIO KENYATTA BARROS. Políticas de Segurança e Defesa da Fronteira Brasileira no Contexto de Integração Regional: os casos das fronteiras Brasil-Paraguai e Brasil-Uruguai. Orientador: Prof. Dr. Alcides Costa Vaz. 2017. 211 p. Dissertação (Programa de Pós-Graduação em Relações Internacionais) - Instituto de Relações Internacionais, Universidade de Brasília, Belo Horizonte, 2017.

GAZETA DO POVO. Lei paraguaia vai legalizar carros roubados. 2007. Disponível em: $<$ https://www.gazetadopovo.com.br/vida-e-cidadania/lei-paraguaia-vai-legalizar-carros-rouba dos-aqqh3u4gxweotllnpjvpifcjy/> Acesso em: 03/05/2020.

MONRAZZOLLI C. JUNG, Real-time brazilian license plate detection and recognition using deep convolutional neural networks, 30th SIB- GRAPI Conference on Graphics, Patterns and Images (SIBGRAPI), pp. 55-62, 2017.

NETZER, Y.; WANG, T., COATES, A.; BISSACO, A.; WU, B. Reading digits in natural images with unsupervised feature learning. In: NIPS workshop on deep learning and unsupervised feature learning. vol. 2011, p. 5 (2011).

REDMON, J. et al. You Only Look Once: Unified, Real-Time Object Detection. IEEE Conference on Computer Vision and Pattern Recognition,2016, pp. 779-788.

SILVA, S, M.; JUNG, C. R. License Plate Detection and Recognition in Unconstrained Scenarios. ECVV, 2018.

ZHANG, H. K.; REN, S. X.; SUN, J. Deep Residual Learning for Image Recognition. In: 2016 IEEE Conference on Computer Vision and Pattern Recognition (CVPR).vol. 4, pp. 770-778. IEEE (jun 2016).

WEINMAN, J.J;, LEARNED-MILLER, E.; HANSON, A.R. Scene text recognition using similarity and a lexicon with sparse belief propagation. IEEE Transactions on pattern analysis and machine intelligence 31(10), 1733-1746 (2009).

ZHANG X, SHEN P, BAI J, et al. (2010) License plate location based on adaboost. In the 2010 IEEE International Conference on Information and Automation. Pp. 1705-1710.

YEE M S, HANZO L.(1999) Radial basis function decision feedback equaliser assisted burst-by- burst adaptive modulation. In Seamless Interconnection for Universal Services. Global Telecommunications Conference. pp. 2183-2187.

WU Y, LIU S, WANG X.(2013) License plate location method based on texture and color. In 2013 IEEE 4th International Conference on Software Engineering and Service Science. pp. 361-364.

KIM S, KIM D, RYU Y, et al. (2002) A robust license-plate extraction method under complex image conditions. In Object recognition supported by user interaction for 
service robots. pp. 216-219.

JIN B, JIA Y. (2011) Algorithmic research of speeding license plate location based on mathematics morphology. In 2011 2nd International Conference on Artificial Intelligence, Management Science and Electronic Commerce. pp. 948-950.

ZHANG F Q, DUAN S K, WANG L D. (2013) Application of memristor cell neural network in license plate location. Computer Science, 2013 (S1): 58-60.

Yu S, Li B, Zhang Q, et al. (2015) A novel license plate location method based on wavelet transform and EMD analysis. Pattern Recognition, 48(1): 114-125.

ABOLGHASEMI V, AHMADYFARD A. (2009) An edge-based color-aided method for license plate detection. Image and Vision Computing, 27(8): 1134-1142.

WAZALWAR D, ORUKLU E, SANIIE J. (2011) Design flow for robust license plate localization In 2011 IEEE International Conference on Electro/Information Technology. pp: $1-5$.

GIRSHICK R, DONAHUE J, DARRELL T, et al. (2014) Rich feature hierarchies for accurate object detection and semantic segmentation. In Proceedings of the IEEE conference on computer vision and pattern recognition. pp. 580-587.

GIRSHICK R.(2015) Fast r-cnn. In Proceedings of the IEEE international conference on computer vision. pp. 1440-1448.

REN S, HE K, GIRSHICK R, et al. (2015) Faster r-cnn: Towards real-time object detection with region proposal networks In Advances in neural information processing systems. pp. 91-99.

REDMON J, DIVVALA S, GIRSHICK R, et al. (2016) You only look once: Unified, real-time object detection. In Proceedings of the IEEE conference on computer vision and pattern recognition. pp. 779-788.

LIU W, ANGUELOV D, ERHAN D, et al. (2016) Ssd: Single shot multibox detector. In European conference on computer vision. pp. 21-37. 